February 18, $2016 \quad$ 3:2 WSPC/INSTRUCTION FILE JETaipei

International Journal of Modern Physics D

(C) World Scientific Publishing Company

\title{
TIME TO MOVE ON?
}

\author{
JOHN ELLIS* \\ Theoretical Particle Physics and Cosmology Group, Physics Department, King's College \\ London, London WC2R 2LS, United Kingdom \\ John.Ellis@cern.ch
}

\begin{abstract}
Cosmology and particle physics have long been dominated by theoretical paradigms: Einstein's general theory of relativity in cosmology and the Standard Model of particle physics. The time may have come for paradigm shifts. Does cosmological inflation require a modification of Einstein's gravity? Have experiments at the LHC discovered a new particle beyond the Standard Model? It is premature to answer these questions, but we theorists can dream about the possibilities.
\end{abstract}

Keywords: Cosmology; Particle Physics; Inflation; LHC.

PACS numbers: 04.25.dg, 04.30.Nk, 04.65.+e, 12.0.Dm, 12.60.Jv. 95.30.Sf, 98.80.Es

KCL-PH-TH/2016-07, LCTS/2016-05, CERN-TH-2016-036

\section{Introduction}

It is a privilege to have been invited to give this talk at the end of a meeting celebrating the centenary of Einstein's general theory of relativity. The organisers undoubtedly expected me to talk about models of cosmological inflation, and most of this talk will indeed be about this subject, and whether it suggests a modification of the minimal Einstein-Hilbert action such as an $R^{2}$ term ${ }^{1}$ or supergravity. ${ }^{[2}$ However, during this conference we received dramatic news from CERN of hints of the possible existence of what might, perhaps be a new massive particle beyond the Standard Model.$^{3}$ In the words of the CERN Director-General, Fabiola Gianotti, we theorists "are allowed to be slightly excited", 4 and I will try to share some of this excitement at the end of my talk ${ }^{a}$.

\section{Cosmological Inflation}

For almost two decades now, the cosmological paradigm has been the $\Lambda$ CDM model, based on the general theory of relativity with the addition of a cosmological constant $\Lambda$. Einstein supposedly regarded the introduction in 1917 of $\Lambda$ as "the biggest

\footnotetext{
*Also Theoretical Physics Department, CERN, CH-1211 Geneva 23, Switzerland.

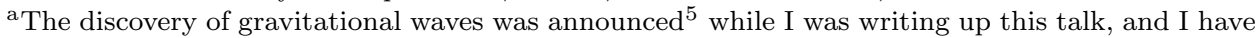
added a note on constraints this discovery imposes on possible modifications of Einstein's theory.
} 
blunder of his life", 6 but nowadays we would beg to differ. Its presence is a possibility allowed by the general principles of relativity, and hence is probably compulsory. The present-day cosmological constant has depressing implications for astronomers in the far future, who will only be able to study our local gravitationally-bound group of galaxies. However, according to the hypothesis of cosmological inflation, ${ }^{7}$ the energy density of the very early universe may have been dominated by an (almost) constant term that would have generated an epoch of (near-) exponential expansion that could explain the great size and age of the universe today and, as an added bonus, have been the origin of the structures seen in the universe today, from clusters of galaxies to Donald Trump.

The most important evidence for cosmological inflation, and constraints on models, come from observations of fluctuations in the cosmic microwave background radiation (CMB), e.g., by the Planck satellite ${ }^{8}$ Together with other cosmological and astrophysical data, these CMB measurements are consistent with the cosmological concordance model with the following energy densities as fractions of that of a universe that is geometrically flat: $\Omega_{\Lambda} \simeq 0.69, \Omega_{\text {matter }} \simeq 0.31$, with most of the latter being cold dark matter (CDM) ${ }^{9}$ I now discuss aspects of the inflationary paradigm and how it is constrained by CMB data.

\subsection{Slow-Roll Inflationary Models}

Most interpretations of the CMB data adopt the framework of slow-roll inflationary models, ${ }^{7}$ in which the near-exponential expansion of the universe would have been driven by the almost constant field energy $V$ of some inflaton scalar field $\phi$ :

$$
\epsilon \equiv \frac{1}{2} M_{P}^{2}\left(\frac{V^{\prime}}{V}\right)^{2}, \eta \equiv M_{P}^{2}\left(\frac{V^{\prime \prime}}{V}\right) \ll 1 .
$$

Quantum fluctuations in $\phi$ would have led to scalar and tensor perturbations in the $\mathrm{CMB}$, and the principal observables are the power-law tilt in the scalar spectrum $n_{s}$, and the tensor-to-scalar ratio $r$, which are given in the slow-roll approximation by

$$
n_{s}=1-6 \epsilon+2 \eta, r=16 \epsilon,
$$

respectively. It is a generic prediction of slow-roll models that the CMB fluctuations should be Gaussian to a very good approximation.

There was great excitement when the BICEP2 experiment reported ${ }^{10}$ the measurement of B-mode polarisation in the $\mathrm{CMB}$, which might have been generated by primordial tensor (quantum gravitational-wave) perturbations. However, these are now thought to consistent with pollution by dust, 11 and a recent combined analysis of BICEP2/Keck array and Planck data yields only an upper limit on $r<0.1, \frac{12}{12}$ as seen in Fig. 1, where we also see that $n_{s} \sim 0.97$. We also see in Fig. 1 that monomial single-field potentials $\propto \phi^{n}$ are disfavoured by the data, whereas the original model of Starobinsky with an $R+R^{2}$ gravitational action ${ }^{1}$ is highly compatible with the 
data, as would be inflation driven by the Higgs field $\frac{13}{13}$ (with a non-mimimal coupling to gravity) or in no-scale supergravity models, ${ }^{2}$ as discussed later. We also note that the experimental restriction on $n_{s}$, in particular, starts to provide an interesting constraint on the number of e-folds of inflationary expansion, $N_{*}$, which is sensitive to how the inflaton decayed into conventional matter, 14 as also discussed later.

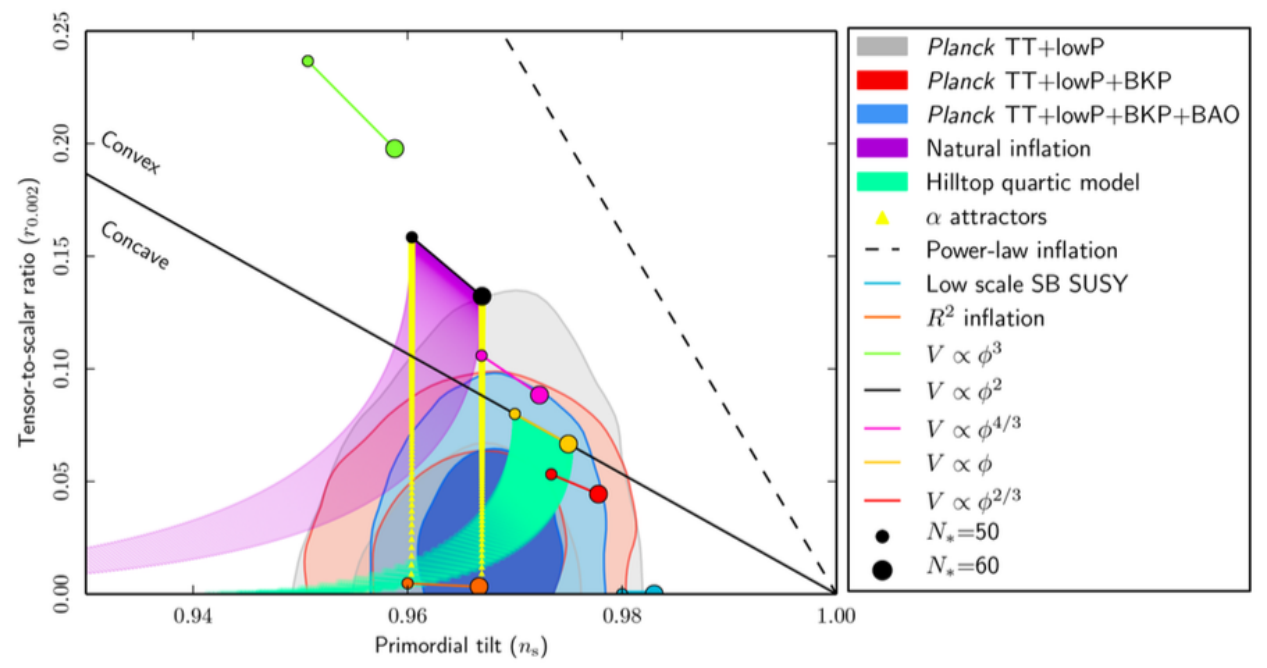

Fig. 1. The marginalised joint 68 and $95 \%$ CL regions for the tilt in the CMB scalar perturbation spectrum, $n_{s}$, and the relative magnitude of the tensor perturbations, $r$, obtained from the Planck 2015 data and their combinations with BICEP2/Keck Array and/or BAO data, ${ }^{12}$ confronted with the predictions of some inflationary models.

\subsection{Challenges for Inflationary Models}

It is already a challenge to write down a simple model of inflation that is compatible with the CMB data, with simple power-law potentials being excluded as seen in Fig. 1. but there are other, deeper challenges.

How can one link inflation to 'low-energy' physics at colliders? Within the Standard Model of particle physics, the only candidate for the inflaton is the Higgs boson, $\frac{13}{, 3}$ but naive extrapolation of Standard Model measurements to high energies indicates that the Higgs potential probably turns negative, $\frac{15}{5}$ wich is unsuitable for inflation.

Can one link inflation to some other well-motivated extension of the Standard Model? For example, could the inflaton be a supersymmetric partner of a righthanded (singlet) neutrino field ${ }^{[16}$ or some sort of axion ${ }^{177}$ ?

Can one link inflation to Planck-scale physics? Specifically, are there plausible inflatons in string theory, or in compactified string models? 


\subsection{The Starobinsky Model}

Let us first review the Starobinsky model,$\frac{1}{1}$ which is based on a non-minimal action for general relativity:

$$
S=\frac{1}{2} \int d^{4} x \sqrt{-g}\left(R+\frac{R^{2}}{6 M^{2}}\right),
$$

where $M$ is some mass parameter that is unknown a priori. It would seem superficially that the action (3) does not contain a scalar inflaton field. However, when one makes a conformal transformation to the Einstein frame ${ }^{[18}$ (in which the dependence on $R$ is purely linear) one find a scalar field model with a characteristic potential:

$$
S=\frac{1}{2} \int d^{4} x \sqrt{\tilde{g}}\left[\tilde{R}+\left(\partial_{\mu} \phi\right)^{2}-\frac{3}{2} M^{2}\left(1-e^{-\sqrt{\frac{2}{3}} \phi}\right)^{2}\right] .
$$

Scalar CMB perturbations were first calculated in this model by ${ }^{19}$ and their small magnitude requires $M \sim 10^{13} \mathrm{GeV} \ll M_{P}$, i.e., a coefficient of the $R^{2}$ term in (3) that is $\gg 1$ in natural units. The value of the scalar tilt $n_{s}$ and the magnitude of the tensor-to-scalar ratio $r \sim 3 \times 10^{-3}$ are highly compatible with the available CMB data, as already seen in Fig. 1 .

\subsection{Higgs Inflation}

William of Occam might have suggested using the (only established) Higgs scalar $h$ as the inflaton, which would require a non-minimal coupling to gravity $\frac{13}{13}$

$$
S=\frac{1}{2} \int d^{4} x \sqrt{-g}\left[-\left(1+\xi h^{2}\right) R+\left(\partial_{\mu} h\right)^{2}-\frac{\lambda}{2}\left(h^{2}-v^{2}\right)^{2}\right],
$$

where $\xi$ is an unknown parameter that must be $\gg 1, \lambda$ is the quartic Higgs selfcoupling and $v$ is the Higgs vev. When $1 \ll \xi \ll 10^{17}$, upon transforming to the Einstein frame one finds

$$
S=\frac{1}{2} \int d^{4} x \sqrt{-\tilde{g}}\left[\tilde{R}+\left(\partial_{\mu} \chi\right)^{2}-\frac{\lambda}{2 \xi^{2}}\left(1+e^{-\sqrt{\frac{2}{3}} \chi}\right)^{-2}\right] .
$$

The resulting effective potential gives successful inflation when $\chi \gg 1$, where the potential is similar to that of the Starobinsky model (though clearly different at small $\chi)$.

The required value of $\xi \sim 15000$, and one may ask how natural that is. However, a more serious practical problem is that one needs $\lambda>1$ for field values beyond the Planck scale, whereas the best available calculations indicate that probably $\lambda<0$ because of renormalization by the top quark $\frac{[15]}{15}$ One accurate calculation indicates that $\lambda$ turns negative at a scale $\Lambda$ :

$\log \frac{\Lambda}{\mathrm{GeV}}=11.3+1.0\left(\frac{M_{h}}{\mathrm{GeV}}-125.66\right)-1.2\left(\frac{m_{t}}{\mathrm{GeV}}-173.10\right)+0.4\left(\frac{\alpha_{3}\left(M_{Z}\right)-0.1184}{0.0007}\right)$.

Inserting the current experimental values of the Higgs mass $M_{h}=125.09 \pm$ $0.24 \mathrm{GeV} \stackrel{201}{\rightleftharpoons}$ the top mass $m_{t}=173.34 \pm 0.27 \pm 0.71 \mathrm{GeV} \stackrel{21]}{2}$ and the strong-interaction 
coupling $\alpha_{3}\left(M_{Z}\right)=0.1177 \pm 0.0013, \frac{22}{22}$ one estimates that the Higgs self-coupling $\lambda$ turns negative at $\ln (\Lambda / \mathrm{GeV})=10.0 \pm 1.0$.

Negative $\lambda$ leads to metastability of the electroweak vacuum and the problem that most of the early universe would have been sucked into the region where the potential is negative. ${ }^{23}$ Physics beyond the Standard Model is needed to avoid these issues, and supersymmetry is one of the possibilities.

\subsection{Inflation Cries out for Supersymmetry and (No-Scale) Supergravity}

There are many other motivations for supersymmetry, and inflation is one of them.24 Models of inflation involve a scalar inflaton field that is elementary, at least at energies $\ll M_{P}$, and obtaining the right magnitude of perturbations requires a mass $\ll M P$ (typically $\sim 10^{13} \mathrm{GeV}$ ) and/or a small inflaton self-coupling $\lambda \ll 1$. Both of these are technically natural in supersymmetric theories.

The first supersymmetric models of inflation were based on global supersymmetry. However, experience (general relativity, the Standard Model) teaches us that the only good symmetry is a local symmetry, and the same should apply to supersymmetry. The local version of supersymmetry necessarily involves local space-time coordinate transformations, and is supergravity. Clearly also, any discussion of early Universe cosmology needs gravity, and supergravity is the way to combine gravity and supersymmetry.

However, there is an issue with generic supergravity models when matter is included, which is that their effective potentials typically have 'holes' with depths $\sim \mathcal{O}\left(M_{P}^{2}\right)$. These make any treatment of cosmology problematic, let alone inflation. There is, however, an exception, namely the class of no-scale supergravity models. 25 The effective potentials in this class of supergravity models have flat directions, and the scalar potential resembles that in globally supersymmetric models, with controlled corrections.

An added motivation for considering no-scale supergravity models is that they arise naturally in compactifications of string theory 26 Therefore, no-scale models of inflation may offer some prospects for making contact with some underlying variant of string theory.

\subsection{No-Scale Supergravity Models of Inflation}

The first no-scale supergravity models of inflation appeared in the $1980 \mathrm{~s}, 27 \mid 28$ largely motivated by the 'unholy' form of the effective potential. This approach was revived in $2013^{2}$ in response to the new, high-precision CMB data from Planck. Supergravity models are characterised by a Hermitian Kähler potential $K$ that specifies the internal geometry of the scalar fields $\phi$, whose kinetic terms are $\left(\partial^{2} K / \partial \phi^{i} \partial \phi_{j}^{*}\right) \partial_{\mu} \phi^{i} \partial^{\mu} \phi_{j}^{*}$. The simplest no-scale inflationary model contains two complex scalar fields:

$$
K=-3 \ln \left(T+T^{*}-\frac{|\phi|^{2}}{3}\right),
$$


and the potential interactions between the scalar fields are characterised by a superpotential $W$, which we assumed to have the form

$$
W=\frac{\mu}{2} \phi^{2}-\frac{\lambda}{3} \phi^{3},
$$

as first studied by Wess and Zumino. ${ }^{[0]}$ We assumed that the field $T$, which could be interpreted as a modulus of string compactification, is fixed by string dynamics: $T=c / 2$, in which case the effective Lagrangian for the inflaton field $\phi$ is

$$
\mathcal{L}_{e f f}=\frac{c}{\left(c-|\phi|^{2} / 3\right)^{2}}\left|\partial_{\mu} \phi\right|^{2}-\frac{\hat{V}}{\left(c-|\phi|^{2} / 3\right)^{2}}: \hat{V}=\left|\frac{\partial W}{\partial \phi}\right|^{2}
$$

We note that the prefactors in the Lagrangian terms in 10 modify the form of the effective potential that one would have obtained in the globally-supersymmetric case, when one transforms to a canonically-normalized inflaton field.

The Wess-Zumino superpotential gives good inflation already in the global case ${ }^{29}$ and we found this to be possible also in its no-scale supergravity incarnation for $\lambda / \mu \sim 1 / 3,2$ as seen in Fig. 2. Indeed, for the specific case $\lambda=\mu / 3$, shown as the black line in Fig. 2 , the effective scalar potential is identical with that in the original Starobinsky model (3, 4).

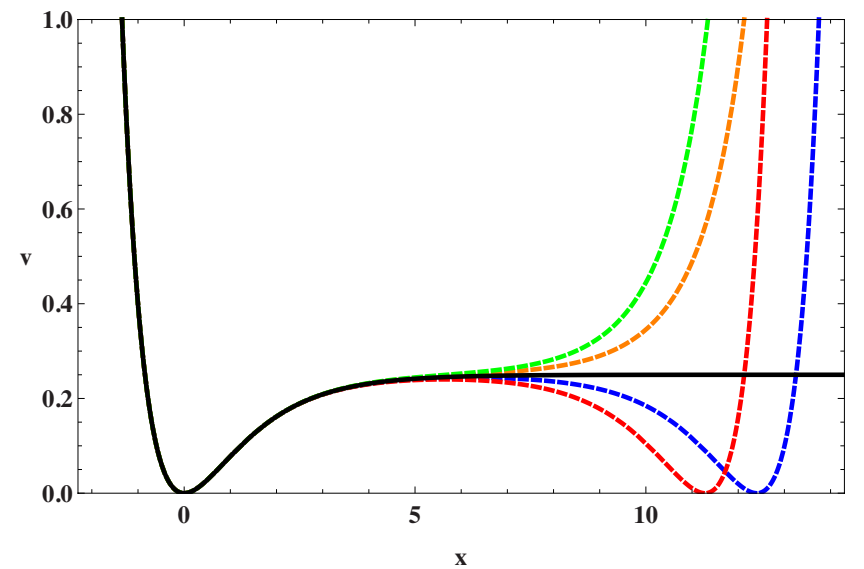

Fig. 2. The inflationary potential $V$ in the Starobinsky $R+R^{2}$ mode ${ }^{11}$ (solid black line) compared with its form in various no-scale model $\$ 2$ (dashed coloured lines).

Looking beyond the Starobinsky limit, one can consider generic potentials of the form $\frac{31}{31}$

$$
V=A\left(1-\delta e^{-B \phi}+\mathcal{O}\left(e^{-2 B \phi}\right)\right) .
$$

In such models, the most prominent inflationary predictions are

$$
n_{s}=1-2 B^{2} \delta e^{B \phi_{*}}, r=8 B^{2} \delta^{2} e^{-2 B \phi_{*}}, N_{*}=\frac{1}{B^{2} \delta} e^{B \phi_{*}},
$$


where $\Phi_{*}$ is the value of the inflaton field that yields $N_{*}$ e-folds of inflation. It is clear from 12 that $n_{s}$ is in a one-to-one relationship with $N_{*}$, and that $r$ may be adjusted, for any fixed value of $N_{*}$, by varying the coefficient $B$ in the exponential in 11 b

As an example how this freedom could be exploited, one can consider models in which the no-scale property is shared among several moduli fields $\underline{31}$

$$
K \ni-\sum_{i} \ln \left(T_{i}+T_{i}^{*}\right): N_{i}>0, \sum_{i} N_{i}=3,
$$

as is characteristic of generic string compactifications. In this case, if the inflaton is identified with $T_{i}$ one has

$$
B=\sqrt{\left(\frac{2}{N_{i}}\right)}, r=\frac{4 N_{i}}{N_{*}^{2}},
$$

with the specific choice of a single modulus with $N_{i}=3$ corresponding to the Starobinsky model (3, 4). A measurement of $r$ could therefore, within this no-scale supergravity framework, provide a unique window on the phenomenology of string compactification.

\subsection{How Many e-folds of Inflation?}

The discussion above shows that the number of e-folds, $N_{*}$, is a key to the phenomenology of inflationary models, and we also see in Fig. 1 that the CMB data are beginning to provide interesting constraints on $N_{*} \cdot \frac{14}{14}$ This can be related to the potential $V_{*}$ at $\phi_{*}$ corresponding to the scale $k_{*}$ :

$$
N_{*}=67-\ln \left(\frac{k_{*}}{a_{0} H_{0}}\right)+\frac{1}{4} \ln \left(\frac{V_{*}^{2}}{M_{P}^{2} \rho_{\text {end }}}\right)+\frac{1-3 w_{\text {int }}}{12\left(1+w_{\text {int }}\right)} \ln \left(\frac{\rho_{\text {reh }}}{\rho_{\text {end }}}\right)-\frac{1}{12} \ln g_{\text {th }},
$$

where $\rho_{\text {end,reh }}$ are the densities at the end of inflation and after reheating, respectively, $w_{\text {int }}$ is the average equation-of-state parameter during inflaton decay and reheating, and $g_{t h}$ is the number of degrees of freedom after reheating.

\subsection{Constraints on Inflaton Decay}

The value of $N_{*}$ and hence $r$, in particular, is sensitive to the inflaton decay rate $\Gamma_{\phi}$, since the ratio $\rho_{\text {reh }} / \rho_{\text {end }} \propto \Gamma_{\phi}^{2}$. Thus a measurement of $r$ can constrain models of inflaton decay. This point is illustrated in Fig. 3 for the Starobinsky-like no-scale supergravity models of inflation whose potentials are shown in Fig. 2 In particular, for the special case $\lambda / \mu=1 / 3$ that reproduces exactly the Starobinsky model, we see that $N_{*} \geq 44$ at the $95 \%$ CL and $\geq 50$ at the $68 \%$ CL. If the dominant inflaton decay is via a trilinear superpotential coupling $y$ into two-body final states, the inflaton decay rate $\Gamma_{\phi}=|y|^{2} / 8 \pi m_{\phi}$, and these constraints on $N_{*}$ correspond to the constraints $y>10^{-16}$ at the $95 \%$ CL and $>10^{-7}$ at the $68 \%$ CL. 14

\footnotetext{
${ }^{\mathrm{b}}$ Similar results are found in the so-called $\alpha$-attractor models ${ }^{32}$
} 


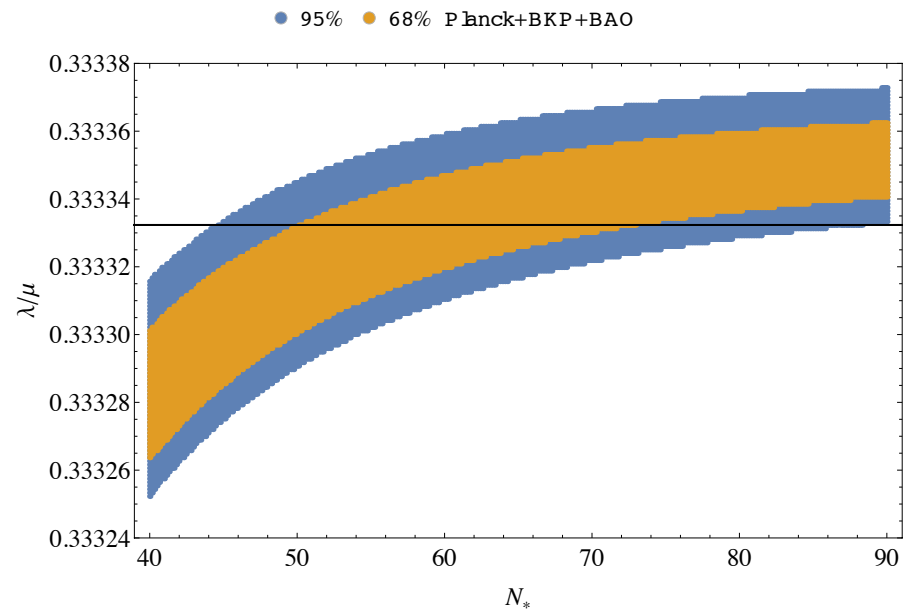

Fig. 3. The $68 \%$ and $95 \%$ CL regions (yellow and blue, respectively) in the $\left(N_{*}, \lambda / \mu\right)$ plane for the no-scale inflationary mode 2 with a matter inflaton field and the Wess-Zumino superpotential 14 The horizontal black line is for $\lambda / \mu=1 / 3$, the value that reproduces the inflationary predictions of the Starobinsky model ${ }^{2}$

These bounds are to be compared with those due to upper limits on $y$ from the production in inflaton decays of gravitinos with mass $m_{3 / 2} \cdot{ }^{[14}$ We find

$$
|y| \lesssim 1.5 \times 10^{-6} \text { for } m_{3 / 2}=6 \mathrm{TeV},
$$

from requiring that long-lived gravitinos do not mess up the success of standard Big-Bang nucleosynthesis (BBN) calculations, weakening for larger $m_{3 / 2}$ and disappearing altogether if the gravitino is sufficiently heavy to decay before BBN. There is also a constraint

$$
|y|<2.7 \times 10^{-5} \times\left(\frac{100 \mathrm{GeV}}{m_{\mathrm{LSP}}}\right)
$$

from the upper limit on the contribution of the lightest supersymmetric particle (LSP) to the cosmological cold dark matter. The latter constraint, in particular, suggests that $|y| \lesssim 10^{-5}$ for LSP masses in the range of a few hundred $\mathrm{GeV}$,

The constraint on the inflaton decay coupling $y$ from the CMB measurement of $r$ is not yet competitive with the other constraints (16, 17), but this can be expected to change in the future.

\section{Meanwhile, back at the LHC}

During 2015, the LHC restarted operations, making collisions at a centre-of-mass energy of $13 \mathrm{TeV}$, much higher than the 7 and $8 \mathrm{TeV}$ during its first run. The two experiments looking for new high-mass physics, ATLAS and CMS, have each accumulated $\sim 3 / \mathrm{fb}$ of high-energy data. They presented their first preliminary data during this conference, and created a sensation. 
Both experiments reported statistical excesses in their $\gamma \gamma$ invariant-mass distributions that could perhaps, maybe, be hints of a possible new particle $X$ with mass $\sim 750 \mathrm{GeV}$ decaying into two photons. $\frac{3}{3}$ The significances in the experiences are only $\sim 3 \sigma$, and are diluted by the 'look-elsewhere' effect that the experiments examined many $\gamma \gamma$ invariant-mass distributions (and many other distributions!), so the overall significance falls far short of the $5-\sigma$ 'gold standard' required to claim discovery of a new particle, particularly one so unexpected. If it exists, the $X$ particle would herald a layer of new physics beyond the Standard Model.

\subsection{Interpretation of the $X(750)$ Hint}

The CMS and ATLAS data suggest a product of cross-section and $\gamma \gamma$ branching ratio $\sim 6 / \mathrm{fb}$, and most analyses assume that it is a scalar or pseudoscalar. The most plausible possibility is that it decays to $\gamma \gamma$ via triangular loops of heavy charged particles, most likely fermions weighing $\gtrsim 400 \mathrm{GeV}{ }^{[33}$ The production of the $X(750)$ is also often thought to be via loops of massive coloured fermions. These could not be a fourth generation of Standard Model particles, and minimal supersymmetric models are also unable to explain the magnitude of the $X$ 'signal'. There would have to be a whole new world out there!

Four simple possibilities for these extra fermions are: i) a single vector-like charge $2 / 3$ quark, ii) an isospin doublet of vector-like charge $2 / 3$ and $-1 / 3$ quarks, iii) an isodoublet and two singlet charge $2 / 3$ and $-1 / 3$ quarks, and iv) a complete vectorlike generation including leptons as well as quarks ${ }^{33} \mathrm{Fig}$. 4 illustrates the possible couplings $\lambda$ in these four models of $X$ to the vector-like fermions (assumed, for simplicity, to be universal) as functions of their masses (also assumed, for simplicity, to be universal) if they are to reproduce the possible 'signal' (assuming that the dominant $X$ decays are those mediated by fermion loops). It is worth noting that model iv) contains a weakly-interacting neutral fermion that is a possible dark matter candidate.

In each of these models, triangular diagrams generate decays of the $X$ particle to other pairs of vector bosons: gluon-gluon, $Z \gamma, Z Z$ and $W^{+} W^{-}$, at rates relative to $\gamma \gamma$ that are easily calculable and depend on the model ${ }^{33}$ It is premature to exclude any of these models, though model ii) is under pressure from searches at the LHC. In addition to these decays, the LHC experiments should also look for the heavy fermions that are required. There will be theoretical and experimental work for a generation, if the $X(750)$ exists. Since the LHC should make many more collisions in 2016, we can expect to know soon whether the $X(750)$ 'signal' is real. In the mean while, we theorists are "allowed to be a little excited" $4\left[\begin{array}{l}\mathrm{C} \\ \mathrm{C}\end{array}\right.$

\footnotetext{
${ }^{c}$ However, we should also remember the words of Laplace, "Plus un fait est extraordinaire, plus il a besoin d'être appuyé de fortes preuves." ("The more extraordinary a claim, the stronger the proof required to support it.")
} 


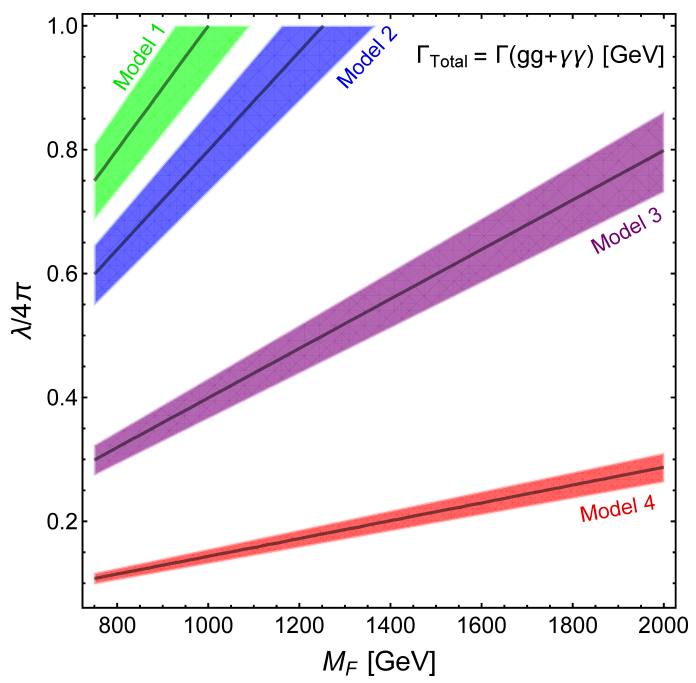

Fig. 4. Values of the vector-like fermion mass $m_{F}$ and coupling $\lambda$ (both assumed to be universal) required models i), ii), iii) and iv) to accommodate the possible $g g \rightarrow \Phi \rightarrow \gamma \gamma$ signal reported by CMS and ATLAS! ${ }^{3}$ The black lines are for the central value of the cross section, $6 \mathrm{fb}$, and the coloured bands represent the $1-\sigma$ uncertainties. Figure from,, 34 adapted from $[33$

\subsection{What's in it for Inflation?}

The appearance of another scalar particle would provide another candidate for the inflaton, and one can consider inflation via a non-minimal $X$ coupling to gravity, just as was discussed for Higgs inflation in Subsection 2.4. The appearance of the $X$ particle could also be used to revive Higgs inflation, or even lead one to consider a two-field model of inflation. If it is real, the $X(750)$ particle could revolutionise inflationary model-building as well as collider physics.

\section{Summary}

As we saw in Section 2, many popular models of inflation postulate some modification of the minimal Einstein-Hilbert action: maybe an $R^{2}$ term as in the Starobinsky, maybe a non-minimal coupling to a scalar field as in Higgs (or $X$ !) inflation, or maybe promote general relativity to supergravity. It is ironic that, 100 years after the proposal of general relativity, at a time when experiment finally confirms its key prediction of gravitational waves, the time may have come to move on from Einstein. As we saw in Section 3, if the possible hint, maybe, of a new particle $X$ with mass $\sim 750 \mathrm{GeV}$ would, if confirmed, require us to move on from the Standard Model of particle physics, potentially also with interesting implications for inflationary model-building. 


\section{Note added}

The discovery of gravitational waves,$[5$ which was announced while this write-up was being completed, is a tremendous vindication of Einstein's theory of general relativity $\stackrel{[36}{ }$ Perhaps this and similar future observations will give us hints how to extend it? In the mean time, the gravitational-wave data already provide some constraints on possible modifications of Einstein's theory, including an upper limit on the graviton mass ${ }^{36} \sim 10^{-22} \mathrm{~g}$, limits on Lorentz violation ${ }^{37}[38$ and the violation of the principle of equivalence, $\frac{39}{3}$ and the possible observation of a $\gamma$-ray flash (if confirmed) would tells us that electromagnetic and gravitational waves travel at the

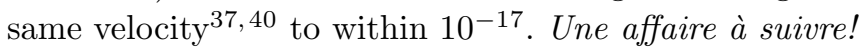

\section{Acknowledgments}

The author was supported partly by the London Centre for Terauniverse Studies (LCTS), using funding from the European Research Council via the Advanced Investigator Grant 26732, and partly by the STFC Grant ST/L000326/1. The author thanks Pisin Chen and his team for their kind hospitality during the Second LeCosPA Symposium, and thanks the Universidad de Antioquia, Medellín, for its hospitality while writing up this talk, using grant FP44842-035-2015 from Colciencias (Colombia).

\section{References}

1. A. A. Starobinsky, Phys. Lett. B 91, 99 (1980).

2. J. Ellis, D. V. Nanopoulos and K. A. Olive, Phys. Rev. Lett. 111 (2013) 111301 [Phys. Rev. Lett. 111 (2013) 12, 129902] arXiv:1305.1247[hep-th]].

3. J. Olsen, CMS physics results from Run 2 presented on Dec. 15th, 2015; M. Kado, ATLAS physics results from Run 2 presented on Dec. 15th, 2015, https://indico.cern.ch/event/442432/; ATLAS Collaboration, ATLAS-CONF2015-081, https://atlas.web.cern.ch/Atlas/GROUPS/PHYSICS/CONFNOTES/ATLASCONF-2015-081/; CMS Collaboration, CMS PAS EXO-15-004, https://cds.cern.ch/ record/2114808/files/EXO-15-004-pas.pdf.

4. F. Gianotti, talk to CERN staff, January 18th, 2016.

5. B. P. Abbott et al. [LIGO Scientific Collaboration and Virgo Collaboration], Phys. Rev. Lett. 116 (2016) 6, 061102 doi:10.1103/PhysRevLett.116.061102 arXiv:1602.03837][gr$\mathrm{qc}]]$.

6. For a discussion of this supposition, see, for example: G. Weinstein, arXiv:1310.1033 [physics.hist-ph].

7. J. Martin, C. Ringeval and V. Vennin, Phys. Dark Univ. 5-6, 75-235 (2014) arXiv:1303.3787 [astro-ph.CO]].

8. P. A. R. Ade et al. [Planck Collaboration], arXiv:1502.02114 [astro-ph.CO].

9. K. A. Olive et al. [Particle Data Group Collaboration], Chin. Phys. C 38 (2014) 090001. doi:10.1088/1674-1137/38/9/090001

10. P. A. R. Ade et al. [BICEP2 Collaboration], Phys. Rev. Lett. 112 (2014) 24, 241101 doi:10.1103/PhysRevLett.112.241101 arXiv:1403.3985 [astro-ph.CO]].

11. R. Adam et al. [Planck Collaboration], Astron. Astrophys. 586 (2016) A133 doi:10.1051/0004-6361/201425034 arXiv:1409.5738 [astro-ph.CO]]. 
12. P. A. R. Ade et al. [BICEP2 and Keck Array Collaborations], arXiv:1510.09217][astroph.CO].

13. F. L. Bezrukov and M. Shaposhnikov, Phys. Lett. B 659, 703 (2008) arXiv:0710.3755 [hep-th]].

14. J. Ellis, M. A. G. Garcia, D. V. Nanopoulos and K. A. Olive, JCAP 1507 (2015) 07, 050 doi:10.1088/1475-7516/2015/07/050 arXiv:1505.06986 [hep-ph]].

15. D. Buttazzo, G. Degrassi, P. P. Giardino, G. F. Giudice, F. Sala, A. Salvio and A. Strumia, JHEP 1312 (2013) 089 arXiv:1307.3536 [hep-ph]].

16. H. Murayama, H. Suzuki, T. Yanagida and J. Yokoyama, Phys. Rev. Lett. 70 (1993) 1912.

17. F. C. Adams, J. R. Bond, K. Freese, J. A. Frieman and A. V. Olinto, Phys. Rev. D 47 (1993) 426 hep-ph/9207245.

18. K. S. Stelle, Gen. Rel. Grav. 9 (1978) 353; B. Whitt, Phys. Lett. B 145 (1984) 176.

19. V.F. Mukhanov and G.V. Chibisov, JETP Lett. 33 (1981) 532.

20. G. Aad et al. [ATLAS and CMS Collaborations], Phys. Rev. Lett. 114 (2015) 191803 doi:10.1103/PhysRevLett.114.191803 arXiv:1503.07589 [hep-ex]].

21. ATLAS, CDF, CMS and D0 Collaborations, arXiv:1403.4427 [hep-ex].

22. D. d'Enterria and P. Z. Skands (eds.), arXiv:1512.05194 [hep-ph].

23. See, for example: M. Fairbairn and R. Hogan, Phys. Rev. Lett. 112 (2014) 201801 doi:10.1103/PhysRevLett.112.201801 arXiv:1403.6786 [hep-ph]]; A. Hook, J. Kearney, B. Shakya and K. M. Zurek, JHEP 1501 (2015) 061 doi:10.1007/JHEP01(2015)061 arXiv:1404.5953 [hep-ph]].

24. J. R. Ellis, D. V. Nanopoulos, K. A. Olive and K. Tamvakis, Phys. Lett. B 118 (1982) 335; R. Holman, P. Ramond and G. G. Ross, Phys. Lett. B 137, 343 (1984).

25. E. Cremmer, S. Ferrara, C. Kounnas and D. V. Nanopoulos, Phys. Lett. B 133 (1983) 61.

26. E. Witten, Phys. Lett. B 155 (1985) 151;

27. A. S. Goncharov and A. D. Linde, Class. Quant. Grav. 1, L75 (1984).

28. J. R. Ellis, K. Enqvist, D. V. Nanopoulos, K. A. Olive and M. Srednicki, Phys. Lett. B 152 (1985) 175 [Erratum-ibid. 156B (1985) 452].

29. D. Croon, J. Ellis and N. E. Mavromatos, Phys. Lett. B 724 (2013) 165 arXiv:1303.6253 [astro-ph.CO]].

30. J. Wess and B. Zumino, Phys. Lett. B 49 (1974) 52. doi:10.1016/0370-2693(74)90578-4

31. J. Ellis, D. V. Nanopoulos and K. A. Olive, JCAP 1310 (2013) 009 arXiv:1307.3537.

32. R. Kallosh and A. Linde, JCAP 1307 (2013) 002 doi:10.1088/1475-7516/2013/07/002 arXiv:1306.5220 [hep-th]].

33. See, for example: J. Ellis, S. A. R. Ellis, J. Quevillon, V. Sanz and T. You, arXiv:1512.05327 [hep-ph] (written during this conference).

34. See, for example: A. Djouadi, J. Ellis, R. Godbole and J. Quevillon, arXiv:1601.03696 [hep-ph].

35. M. Dhuria and G. Goswami, arXiv:1512.06782 [hep-ph]; Y. Hamada, T. Noumi, S. Sun and G. Shiu, arXiv:1512.08984[hep-ph]; S. F. Ge, H. J. He, J. Ren and Z. Z. Xianyu, arXiv:1602.01801 [hep-ph].

36. B. P. Abbott et al. [LIGO Scientific and Virgo Collaborations], arXiv:1602.03841 [grqc].

37. J. Ellis, N. E. Mavromatos and D. V. Nanopoulos, arXiv:1602.04764 [gr-qc].

38. A. Kostelecky and M. Mewes, arXiv:1602.04782 [gr-qc].

39. E. O. Kahya and S. Desai, arXiv:1602.04779 [gr-qc].

40. X. Li, F. W. Zhang, Q. Yuan, Z. P. Jin, Y. Z. Fan, S. M. Liu and D. M. Wei, arXiv:1602.04460 [astro-ph.HE]. 